\title{
Melatonin levels in follicular fluid as markers for IVF outcomes and predicting ovarian reserve
}

\author{
Jing Tong ${ }^{1,2}$, Shile Sheng ${ }^{3}$, Yun Sun ${ }^{1,2}$, Huihui $\mathrm{Li}^{1,2,4}$, Wei-Ping $\mathrm{Li}^{1,2}$, Cong Zhang ${ }^{1,2,5}$ \\ and Zi-Jiang Chen 1,2,6,7,8,9 \\ ${ }^{1}$ Center for Reproductive Medicine, Ren Ji Hospital, School of Medicine, Shanghai Jiao Tong University, \\ Shanghai, China, ${ }^{2}$ Shanghai Key Laboratory for Assisted Reproduction and Reproductive Genetics, \\ Shanghai, China, ${ }^{3}$ Department of Nuclear Medicine, Ren Ji Hospital, School of Medicine, Shanghai Jiao Tong \\ University, Shanghai, China, ${ }^{4}$ Department of Obstetrics and Gynecology, Center for Reproductive Medicine, \\ Qilu Hospital of Shandong University, Jinan, China, ${ }^{5}$ Key Laboratory of Animal Resistance Research, College of Life \\ Science, Shandong Normal University, Ji'nan, Shandong, China, ${ }^{6}$ Center for Reproductive Medicine, \\ Shandong Provincial Hospital, Shandong University, Jinan, China, ${ }^{7}$ National Research Center for Assisted \\ Reproductive Technology and Reproductive Genetics, Jinan, China, ${ }^{8}$ The Key Laboratory for Reproductive \\ Endocrinology of Ministry of Education, Jinan, China and ${ }^{9}$ Shandong Provincial Key Laboratory of Reproductive \\ Medicine, Jinan, China
}

Correspondence should be addressed to C Zhang or W-P Li or Z-J Chen; Email: zhangxinyunlife@163.com or liweiping@renji. com or chenzijiang@hotmail.com

\begin{abstract}
Good-quality oocytes are critical for the success of in vitro fertilization (IVF), but, to date, there is no marker of ovarian reserve available that can accurately predict oocyte quality. Melatonin exerts its antioxidant actions as a strong radical scavenger that might affect oocyte quality directly as it is the most potent antioxidant in follicular fluid. To investigate the precise role of endogenous melatonin in IVF outcomes, we recruited 61 women undergoing treatment cycles of IVF or intracytoplasmic sperm injection (ICSI) procedures and classified them into three groups according to their response to ovarian stimulation. Follicular fluid was collected to assess melatonin levels using a direct RIA method. We found good correlations between melatonin levels in follicular fluid with age, anti-Müllerian hormone (AMH) and baseline follicle-stimulating hormone (bFSH), all of which have been used to predict ovarian reserve. Furthermore, as melatonin levels correlated to IVF outcomes, higher numbers of oocytes were collected from patients with higher melatonin levels and consequently the number of oocytes fertilized, zygotes cleaved, top quality embryos on D3, blastocysts obtained and embryos suitable for transplantation was higher. The blastocyst rate increased in concert with the melatonin levels across the gradient between the poor response group and the high response group. These results demonstrated that the melatonin levels in follicular fluid is associated with both the quantity and quality of oocytes and can predict IVF outcomes as well making them highly relevant biochemical markers of ovarian reserve.

Reproduction (2017) 153 443-451
\end{abstract}

\section{Introduction}

Infertility is defined as the inability to achieve pregnancy within a year of unprotected intercourse (Rad et al. 2015). It is not only a medical concern, but also a social issue with increasing prevalence in both developed and developing countries. Over the past 35 years, IVF as an effective treatment for infertility has become widely accepted. However, poor oocyte quality remains one of the most intractable causes of the failure of IVF procedures (Tamura et al. 2009). The ovarian reserve, which indicates female's reproductive potential, is associated with both oocyte quantity and quality. Agerelated quality decrease in the ovary reserve occurs as a result of two processes: cellular malfunction caused by the accumulation of reactive oxygen species (ROS) in cells (Stadtman 2002) and compromised antioxidant defense mechanisms. Furthermore, not only aging itself but also standard IVF procedures such as superovulation, oocyte cryopreservation and freezing of surplus embryos could lead to the accumulation of ROS (Ali et al. 2003, Chao et al. 2005, Tamura et al. 2012).

It is acknowledged that excess ROS in follicular fluid will deteriorate oocyte quality (Goud et al. 2008) by inducing apoptosis of oocyte and granulosa cells (Zhang et al. 2006). These harmful effects of ROS can be inhibited by intracellular antioxidant systems such as 
glutathione (GSH) and ascorbic acid (Wang et al. 2002), and the balance between ROS production and their detoxification therefore becomes essential for the quality of oocytes. Melatonin (N-acetyl-5-methoxytryptamine) is famous as a potent free radical scavenger and a broad-spectrum antioxidant (Manchester et al. 2015). Melatonin, which is an ubiquitously distributed and functionally diverse molecule, can modulate the physiology and molecular biology of cells via various mechanisms (Reiter et al. 2010). In humans, melatonin is the principle secretory product of the pineal gland and is involved in many of the physiology manifestations (Chen et al. 2011). Although investigators observed the phenomenon of melatonin-exerted effects on mammalian reproductive activities decades ago (Reiter et al. 2009), contradictory reports on the effect of melatonin on the reproductive axis exist. Early studies have shown that melatonin can influence the reproductive function at the level of the brain and pituitary by activating the receptor sites within the hypothalamic-pituitary-gonadal axis (Malpaux et al. 2001). However, other studies have demonstrated that melatonin did not have a direct influence on GnRH neurons (Kauffman et al. 2007).

As for oocytes, they are protected and nourished by the surrounding follicular fluid that is regarded as a biological 'window' that reflects the metabolic and hormonal processes of oocytes and granulosa cells (Wiener-Megnazi et al. 2004), and the actual environment in which these cells exist. Interestingly, a high concentration of melatonin, almost threefold higher than that in serum (Brzezinski et al. 1987), has been detected in human follicular fluid (Reiter et al. 2014). These concentrations are maintained presumably not only by active ovarian uptake (Tamura et al. 2008) from the general circulation but also by ovarian synthesis (Itoh et al. 1999). Melatonin synthesized by ovarian cells, such as rat oocytes (Sakaguchi et al. 2012), bovine cumulus cells (Reiter et al. 2013) and human granulosa cells (Reiter et al. 2009), would then diffuse into follicular fluid. As melatonin exerts its antioxidant actions as a direct free radical scavenger and by regulation of gene transcription for antioxidative enzymes (Rodriguez et al. 2004), melatonin in follicular fluid can protect the oocyte from oxidative stress (Reiter et al. 2014) and may act as a direct modulator of oocyte quality.

Currently, much attention is paid on the beneficial impacts of supplement of melatonin on culture medium in animal experiments such as in vitro oocyte protection (Kang et al. 2009, Nakano et al. 2012) and embryo development (Dehghani-Mohammadabadi et al. 2014, Niknafs et al. 2014). However, the precise role of endogenous melatonin in human follicular fluid, and, in particular, a focus on its association with the IVF outcomes for infertile females remains to be determined.

The goal of the present study was to investigate the roles of intrafollicular melatonin. We designed a retrospective study to verify the roles of endogenous melatonin with a focus on the relationship between melatonin levels and other clinical parameters linked to ovarian reserve and the IVF outcomes.

\section{Materials and methods}

\section{Study population}

Participants were recruited from the Center for Reproductive Medicine, Ren Ji Hospital, School of Medicine, Shanghai Jiao Tong University between June 2015 and August 2015. This research was conducted with patients after informed consent according to the guidelines of the Ministry of Public Health of China (MPH) and was approved by the Reproductive Ethics Committee of the Ren Ji Hospital. Sixty-one women undergoing a treatment cycle of IVF or intracytoplasmic sperm injection (ICSI) were retrospectively studied. Patients were nonsmokers and were free from major medical illness. Patients with a congenital uterine anomaly, ovarian tumors or chronic use of any medication were excluded. All patients presented with tubal, male or mixed factor infertility.

The corresponding demographic and related medical data of the participants, including age, type and duration of infertility, and body mass index (BMI) were collected. Serum levels of baseline follicle-stimulating hormone (bFSH) before stimulation, baseline luteinizing hormone (bLH), $\mathrm{E}_{2}$ and antiMüllerian hormone (AMH) were also measured on the second to the fifth day of the previous menstrual cycle.

\section{Stimulation protocols}

Controlled ovarian hyperstimulation is a principal step of IVF therapy, and many stimulation protocols are available for controlled ovarian hyperstimulation in IVF, including the option of long agonist protocol, short agonist protocol, antagonist protocol or minimal stimulation protocol. The doses of gonadotropin mixtures given to each patient are usually adapted, depending on clinical experience. The decision is based on the benefits and shortcomings of each treatment option, and most importantly on the patients' response to ovarian stimulation. FSH (Fostimo, Genevrier, SophiaAntipolis, France; Gonal-F, Merck Serono, Lyon, France; Puregon, MSD, Courbevoie, France) or human menopausal gonadotropin (Menopur, Ferring SAS, St Prex, Switzerland) were used for hyperstimulation. Follicle development was monitored by ultrasound scans from the day of stimulation and every two or three days until the day of human chorionic gonadotropin (hCG) application. The levels of serum $\mathrm{E}_{2}, \mathrm{FSH}$, $\mathrm{LH}$ and progesterone $(\mathrm{P})$ were also detected the same time. When the leading follicle reached $20 \mathrm{~mm}$ in mean diameter with a serum $E_{2}$ level of $200-300 \mathrm{pg} / \mathrm{mL}$ per mature follicle (greater than $14 \mathrm{~mm}$ ), the patients received hCG (Ovitrelle, Merck Serono, Lyon, France; Lizhu, Zhuhai, China) or triptorelin acetate (Decapeptyl, Ferring, Kiel, Germany; Decapeptyl, Ipsen, Paris, France). Ultrasound-guided oocyte collection was performed 33-36 h later. Type and dose of hCG or triptorelin acetate on each patient were selected by the physicians according to the plasma $\mathrm{E}_{2}$ levels on the day of hCG administration and the number of mature oocytes. 


\section{IVF treatment and embryo assessment}

Classical IVF or ICSI was performed according to the clinical indication. ICSI was performed only in the cases of a severe male factor such as oligoasthenoteratozoospermia, obstructive and non-obstructive azoospermia or previous fertilization failure. The day of oocyte retrieval was considered as day 0 . Fertilization was confirmed at $16-18 \mathrm{~h}$ after insemination by the presence of two pronuclei. Cleavage-stage embryos were characterized by four grades depending on their morphological appearance (blastomere number, size and cytoplasmic fragmentation) following Balaban et al. (2001). According to our laboratory strategy, no more than four 'top' (excellent or good) quality cleavage-stage embryos were manipulated on D3, among them, two were transferred in a fresh cycle and the other two were cryopreserved; however, if the fresh embryo transfer was canceled, all the four were cryopreserved for future transfer. A maximum of two embryos were transferred each time. Supernumerary embryos were cultured to day 5 or day 6. Blastocyst assessment was evaluated according to the system of Gardener and Schoolcraft (Gardner et al. 2000). Only expanded blastocysts with at least several inner cell mass cells and some trophectoderm cells were vitrified.

\section{Grouping criteria}

In response to ovarian stimulation, all participants were subgrouped as poor, normal and high ovarian response. Poor ovarian response is defined by the presence of two of the following three features: ( 1 ) advanced maternal age ( $\geq 40$ years) or any other risk factor for poor response, (2) previous characterized poorly responsive cycles $(\leq 3$ oocytes retrieved with a conventional stimulation protocol), (3) an abnormal ovarian reserve test (i.e. antral follicle count $<5-7$ follicles or $\mathrm{AMH}<0.5-1.1 \mathrm{ng} / \mathrm{mL}$ ), according to the 'Bologna criteria' (Ferraretti et al. 2011). The normal and high ovarian response definition is still debated. In our study, we took the agreement (Qiao et al. 2015) of 'Society of Reproductive Medicine Study Groups of the Chinese Medical Association' for reference. Normal ovarian response was defined as follows: (1) age $<35$ years, (2) normal ovarian reserve test $(\mathrm{bFSH}<10 \mathrm{IU} / \mathrm{mL}$ and $1-1.4 \mathrm{ng} / \mathrm{mL}<\mathrm{AMH}<3.5-4.0 \mathrm{ng} / \mathrm{mL}$ ) and (3) no history of cycle cancelation because of poor or high ovarian response. High ovarian response was defined as follows: (1) the number of oocytes retrieved is more than 15 or cycle cancelation is recommended when overabundance mature follicles are observed, (2) ovarian hyperstimulation syndrome observed after hormone treatment, (3) the number of follicles $(>12-14 \mathrm{~mm}$ ) is more than 20 or (4) the serum $E_{2}$ level is higher than $5000 \mathrm{pg} / \mathrm{mL}$ in controlled ovarian hyperstimulation.

\section{Collection of follicular fluid}

Follicular fluid (without any diluting fluid or blood contamination) from the first leading dominant follicle (diameter 19-24 mm) per patient was collected by follicle puncture under transvaginal ultrasound guidance. All follicular aspirations were performed between 08:00 h and 11:00 h. After oocyte isolation, the fluid was centrifuged at $1000 \mathrm{~g}$ for $20 \mathrm{~min}$ at $0^{\circ} \mathrm{C}$ to remove cellular components. Then, the supernatants were collected and stored at $-20^{\circ} \mathrm{C}$ for subsequent assay on the same day within 2 months. All procedures were performed under protection from light.

\section{Melatonin radioimmunoassay (RIA)}

Intrafollicular concentrations of melatonin were measured by ${ }^{125}$ I-Radioimmunoassay using a RIA kit from DIAsource ImmunoAssays S.A. (Belgium) followed by the fundamental principle of RIA, involving competition between a radioactive and a non-radioactive antigen for a fixed number of antibodybinding sites. The measuring range for melatonin was 2.3$1000 \mathrm{pg} / \mathrm{mL}$. The intra-assay coefficient of variation was 9.7-13.4\% and inter-assay co coefficient of variation 8.0$13.3 \%$. The melatonin levels in follicular fluid were calculated by multiplying the melatonin value in single sample of follicular fluid by the number of oocytes retrieved from each person.

\section{Statistical analysis}

All statistical analyses were performed using SPSS software (version 19.0, IBM Corporation, USA). Based on the Kolmogorov-Smirnov test and the Shapiro-Wilk test, normal distribution for all the parameters could not be assumed. Therefore, in descriptive statistics, median values and interquartile ranges (IQR) are given additionally to mean and S.D. Nonparametric test procedures were used for statistical evaluation of the data.

Kruskal-Wallis test was performed to analyze differences in parameter levels between patients with poor, normal and high ovarian response to ovarian stimulation. In the case of significance, pairwise comparisons between different groups were performed with the Mann-Whitney $U$ test. Association between the melatonin levels in follicular fluid and other parameters was measured and tested by Spearman rank correlation coefficients $\left(r_{s}\right)$. Qualitative data were compared between groups according to the chi-squared test. Statistical significance was set at $P<0.05$.

\section{Results}

\section{Baseline characteristics of patients and the melatonin levels in follicular fluid on the day of oocyte retrieval}

In total, 61 women (23-45 years old) were enrolled in this study and divided into three groups: poor, normal and high ovarian response according to their response to ovarian stimulation. The characteristics of the patients in the subgroups were described in Table 1. The results showed that, as expected, age, bFSH and bE2, which could predict ovarian reserve negatively, were the highest in poor ovarian response group, and the differences were quite marked. While $\mathrm{AMH}$, another critical marker of ovarian reserve, showed an opposite tendency among the groups, the 


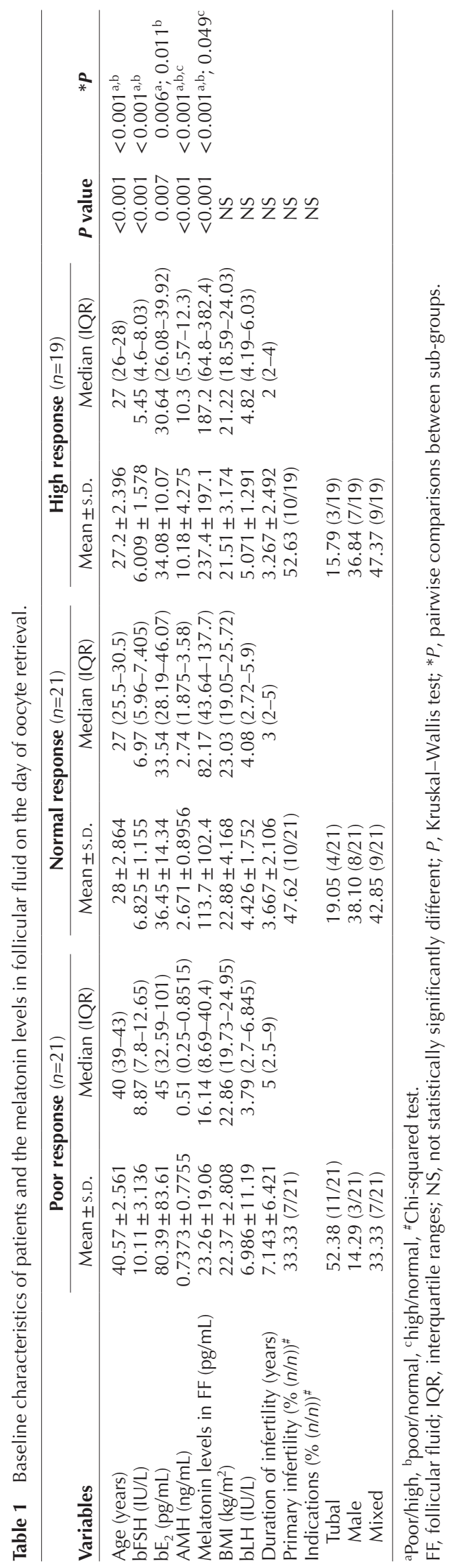

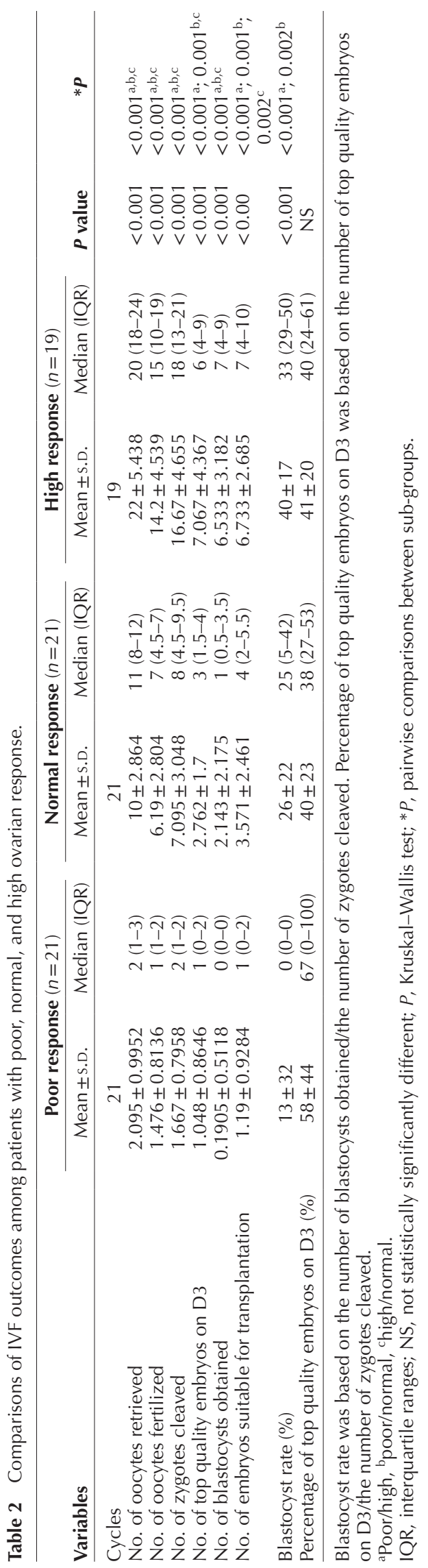

www.reproduction-online.org 
high response group had the maximum AMH levels, whereas the poor ovarian response group had the lowest $(P=0.000)$. Interestingly, the melatonin levels in follicular fluid on the day of oocyte retrieval also showed a statistically significant difference between groups $(P=0.000)$, the values were $23.26 \pm 19.06(\mathrm{pg} /$ $\mathrm{mL})$ for the poor response group, $113.7 \pm 102.4(\mathrm{pg} /$ $\mathrm{mL}$ ) for the normal response group and $237.4 \pm 197.1$ $(\mathrm{pg} / \mathrm{mL})$ for the high response group. Other baseline characteristics such as BMI, bLH, melatonin in single sample of follicular fluid, duration of infertility, primary infertility and indications for infertility including tubal, male and mixed showed no significant differences among the three groups.

\section{Comparisons of IVF outcomes among patients with poor, normal and high ovarian response}

When comparing the IVF outcomes among groups, we observed that the patients in poor ovarian response group who had the lowest melatonin levels in follicular fluid had poorest outcomes of IVF. As showed in Table 2, the criteria of IVF outcomes such as the number of oocytes retrieved, oocytes fertilized, zygotes cleaved, top quality embryos on D3, blastocysts obtained and embryos suitable for transplantation, as well as the rate of development to blastocyst increased significantly from patients with the poor ovarian response. All parameters were greater in the normal response group and reached a maximum in patients with elevated response. The melatonin's concentration increased at the same time among the groups.

Additionally, we did the paired comparisons among the three groups. We observed there were differences in the number of oocytes retrieved, oocytes fertilized, zygotes cleaved, top quality embryos on D3, blastocysts obtained and embryos suitable for transplantation among the three groups (Table 2). As for the blastocyst rate, there was a difference between poor and normal and poor and high ovarian response groups (Table 2). Although the percentage of top quality embryos on D3 showed no significant difference among the three groups (Table 2).

\section{Melatonin levels in follicular fluid on the day of oocyte retrieval are highly relevant markers of ovarian reserve}

Analysis of non-normal distributed values of melatonin and $\mathrm{AMH}$ levels, showed a positive correlation (Spearman-Rho $r_{\mathrm{s}}=0.593, \quad P<0.001$ ) (Fig. 1A). Additionally, we found a negative correlation between the melatonin levels and other ovarian reserve parameters, including age $\left(r_{\mathrm{s}}=-0.632, P<0.001\right)$ (Fig. 1B) and bFSH $\left(r_{\mathrm{s}}=-0.507, P<0.001\right)$ (Fig. 1C). Moreover, we analyzed the correlation of the melatonin levels and ovarian stimulation parameters to explore the influence of stimulation protocols on the melatonin levels in follicular fluid. We identified the relationship between the melatonin levels and stimulation characteristics in patients $(n=41)$ who used the standard long agonist protocol, short agonist protocol and antagonist protocol, as summarized in Table 3. We observed that the melatonin levels had a positive correlation with plasma $\mathrm{E}_{2}$ and progesterone levels on the day of hCG administration (dhCG), their correlation coefficients were $r_{\mathrm{s}}=0.636$ and $P<0.001$ for $\mathrm{E}_{2}$-dhCG and $r_{s}=0.473$ and $P<0.001$ for P-dhCG; at the same time, the melatonin levels had a negative correlation with LH-dhCG $\left(r_{\mathrm{s}}=-0.382, \quad P=0.002\right)$. However, there were no significant relationships between the melatonin levels and serum FSH levels on the day of hCG administration (FSH-dhCG), duration of stimulation, the initial dose and total dose of gonadotropin and endometrial thickness (mm) (Table 3).

\section{Melatonin levels in follicular fluid on the day of oocyte retrieval can predict IVF outcomes}

To further explore the function of melatonin in the follicular fluid on the day of oocyte retrieval in predicting IVF outcomes, we identified the correlation of the melatonin levels in follicular fluid and IVF outcomes. We observed that the melatonin levels had a significant positive correlation with IVF outcomes parameters (Table 4), including number of oocytes retrieved $\left(r_{\mathrm{s}}=0.67, \quad P<0.001\right)$, oocytes fertilized $\left(r_{\mathrm{s}}=0.653\right.$, $P<0.001)$, zygotes cleaved $\left(r_{\mathrm{s}}=0.646, \quad P<0.001\right)$,
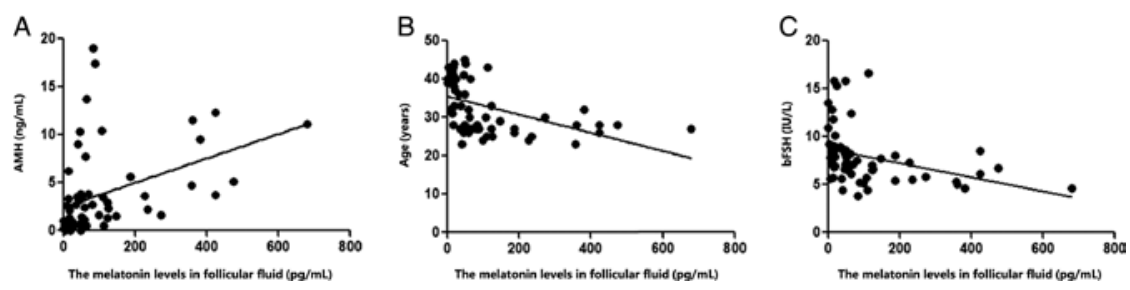

Figure 1 Correlation between the melatonin levels in FF on the day of oocyte retrieval and AMH, age and bFSH levels $(n=61)$. (A) A significant positive correlation between the melatonin levels in follicular fluid and AMH level (Spearman-Rho $r_{\mathrm{s}}=0.593, P<0.001$ ). (B) A significant negative correlation between the melatonin levels in follicular fluid and female age (Spearman-Rho $r_{\mathrm{s}}=-0.632, P<0.001$ ). (C) A significant negative correlation between the melatonin levels in follicular fluid and bFSH level (Spearman-Rho $r_{\mathrm{s}}=-0.507, P<0.001$ ). 
Table 3 Correlation between the melatonin levels in follicular fluid $(\mathrm{pg} / \mathrm{mL})$ and ovarian stimulation parameters in patients $(n=41)$ who used the standard long agonist protocol, short agonist protocol and antagonist protocol.

\begin{tabular}{lcc}
\hline Parameters & $\begin{array}{c}\text { The melatonin levels in } \\
\text { follicular fluid }(\mathrm{pg} / \mathrm{mL}) *\end{array}$ & $\boldsymbol{P}$ value \\
\hline $\mathrm{E}_{2}$-dhCG $(\mathrm{pg} / \mathrm{mL})$ & 0.636 & $<0.001$ \\
P-dhCG $(\mathrm{ng} / \mathrm{mL})$ & 0.473 & $<0.001$ \\
LH-dhCG $(\mathrm{IU} / \mathrm{L})$ & -0.382 & 0.002 \\
FSH-dhCG $(\mathrm{IU} / \mathrm{L})$ & -0.007 & $\mathrm{NS}$ \\
Duration of stimulation & 0.143 & $\mathrm{NS}$ \\
$\quad$ (days) & & $\mathrm{NS}$ \\
Total dose of Gn (IU) & -0.077 & $\mathrm{NS}$ \\
Initial dose of Gn $(\mathrm{IU})$ & -0.297 & $\mathrm{NS}$ \\
Endometrial thickness $(\mathrm{mm})$ & 0.103 & \\
\hline
\end{tabular}

*Spearman rank correlation coefficients $\left(r_{\mathrm{s}}\right)$.

$E_{2}$-dhCG, serum $E_{2}$ levels on the day of hCG administration; FSH-dhCG, serum FSH levels on the day of hCG administration; LH-dhCG, serum LH levels on the day of hCG administration; NS, not statistically significantly different; P-dhCG, serum P levels on the day of hCG administration.

top quality embryos on D3 $\left(r_{\mathrm{s}}=0.587, P<0.001\right)$, embryos cultured $\left(r_{\mathrm{s}}=0.559, \quad P<0.001\right)$, blastocysts obtained $\left(r_{\mathrm{s}}=0.533, P<0.001\right)$, embryos suitable for transplantation $\left(r_{\mathrm{s}}=0.607, P<0.001\right)$ and blastocyst rate $\left(r_{\mathrm{s}}=0.36, P=0.005\right)$. However, we found the melatonin levels in follicular fluid had no significant correlation with percentage of top quality embryos on D3. Given the results described previously, the melatonin levels in follicular fluid have a significant positive correlation with IVF outcomes.

\section{Discussion}

In this study, we determined the melatonin levels in follicular fluid and investigated their relationships with the clinical parameters and the outcomes of IVF. The results demonstrated that the melatonin levels in the follicular fluid displayed good correlations with

Table 4 Correlation of the melatonin levels in follicular fluid on the day of oocyte retrieval and IVF outcomes parameters $(n=61)$.

\begin{tabular}{lcc}
\hline Parameters & $\begin{array}{c}\text { The melatonin levels in } \\
\text { follicular fluid* }\end{array}$ & $\boldsymbol{P}$ value \\
\hline $\begin{array}{l}\text { No. of oocytes retrieved } \\
\text { No. of oocytes fertilized }\end{array}$ & 0.67 & $<0.001$ \\
$\begin{array}{l}\text { No. of zygotes cleaved } \\
\text { No. of top quality embryos }\end{array}$ & 0.653 & $<0.001$ \\
$\quad$ on D3 & 0.646 & $<0.001$ \\
$\begin{array}{l}\text { No. of embryos cultured } \\
\text { No. of blastocysts obtained }\end{array}$ & 0.587 & $<0.001$ \\
$\begin{array}{l}\text { No. of embryos suitable for } \\
\text { transplantation }\end{array}$ & 0.559 & $<0.001$ \\
$\begin{array}{l}\text { Blastocyst rate (\%) } \\
\text { Percentage of top quality } \\
\text { embryos on D3 (\%) }\end{array}$ & 0.607 & $<0.001$ \\
& 0.36 & $<0.001$ \\
\end{tabular}

* Spearman rank correlation coefficients $\left(r_{\mathrm{s}}\right)$.

Blastocyst rate was based on the number of blastocysts obtained/the number of zygotes cleaved. Percentage of top quality embryos on D3 was based on the number of top quality embryos on D3/the number of zygotes cleaved.

NS, not statistically significantly different. age, $\mathrm{AMH}$ and $\mathrm{bFSH}$, which are reliable predictors of ovarian reserve. Furthermore, melatonin levels were crucial to IVF outcomes, patients with higher melatonin levels retrieved more oocytes, and the number of oocytes fertilized, zygotes cleaved, top quality embryos on D3, blastocysts obtained and embryos suitable for transplantation as well as blastocyst rate increased significantly together with the melatonin levels from the poor response group to high response group. Thus, melatonin levels in the follicular fluid can act as a good predictor of ovarian reserve as well as IVF outcomes.

The ovarian reserve associated with both quantity and quality of oocytes indicates female's reproductive potential and reflects the outcomes of IVF as a result. Although IVF treatment outcomes are highly variable and difficult to predict, the ovarian reserve is now routinely tested in women undergoing IVF cycles. This pretreatment assessment of ovarian reserve allows individual prediction of ovarian responsiveness and modification of individual treatment protocol in an attempt to maximize IVF success rates. Both spontaneous and treatment-induced pregnancy rates decline with women aging as a result of both a quantitative and a qualitative reduction of the primordial follicle pool (Bancsi et al. 2002). Unfortunately, there is no marker of ovarian reserve available that can accurately predict oocyte quality (Aydın et al. 2015). AMH as well as bFSH is by now presented as a good serum marker of ovarian reserve. Nevertheless, AMH is not related to embryo quality; its predictive capacity is too limited to be of clinical value (Smeenk et al. 2007). An elevated bFSH reflects a quantitative rather than qualitative decline of the ovarian reserve (Abdalla \& Thum 2004). So neither of them can be used to predict oocyte quality.

The follicular fluid microenvironment is crucial for oocytes quality as oocytes are protected and nourished by follicular fluid in vivo. Excess ROS in follicular fluid caused by aging or IVF procedures will deteriorate oocyte quality (Goud et al. 2008); therefore, it is vital to get a balance between ROS production and their detoxification for the quality of oocytes. As a broadspectrum antioxidant and a potent free-radical scavenger, melatonin can collaborate against excess ROS within cells by causing a cascade reaction (Tan et al. 2007) and modulating antioxidant enzymes gene transcription (Tomás-Zapico \& Coto-Montes 2005). Granulosa cells play a crucial role in follicular steroidogenesis and are involved in establishing an essential microenvironment for the oocyte. Evidence indicated that melatonin acted as an antioxidant to protect the integrity of granulosa cells and influences the production of progesterone and estradiol in porcine (He et al. 2016). So we assume that melatonin as the primary antioxidant in follicular fluid could protect oocytes from being oxidatively abused by free-radical damage through quenching ROS (Wang et al. 2013) and has a positive impact on oocyte quality as a consequence. 
Our results showed a good correlation between the melatonin levels in follicular fluid and $\mathrm{AMH}$. This factor is synthesized exclusively by granulosa cells of secondary, preantral and small antral follicles (Weenee et al. 2004) the same cells where melatonin is produced in the ovary. The amount of melatonin produced by human pineal gland diminishes with advancing age (Tan et al. 2007). Although the mechanism of decreasing melatonin levels with aging is not yet clearly understood, the explanation appears to be (1) increased calcareous deposits in the pineal gland; (2) decreased $\beta$-adrenergic receptors in the pinealocyte membranes or the poor response to norepinephrine; (3) the decreased $\mathrm{N}$-acetyltransferase in the pineal gland with age; (4) progressive alterations in the concentration of melatonin $\alpha 1$-receptors in hypothalamus and (5) increased clearance of melatonin (Touitou 2001). The same tendency was found in the concentration of melatonin in the follicular fluid according to our findings. This finding of an age-related decrease of melatonin is in general agreement with the research on serum melatonin (Waldhauser et al. 1988) and on urinary melatonin (Vakkuri et al. 1996). Furthermore, our results showed a negative correlation between the melatonin levels in follicular fluid and serum bFSH level, which was in agreement with previous studies (Walecka-Kapica et al. 2015). As age, AMH and bFSH reflect the ovarian reserve, we conclude that the melatonin levels in the follicular fluid are markers of ovarian reserve and diminish with advancing age, possibly because of the dysfunction of granulosa cells and pineal gland.

Melatonin levels are highly related to IVF outcomes, patients with higher melatonin levels yielded more oocytes, and the number of oocytes fertilized, zygotes cleaved, top quality embryos on D3, blastocysts obtained, embryos suitable for transplantation as well as blastocyst rate, increased significantly from the poor response group to high response group. The blastocyst rate is an indicator of oocyte developmental competence and of oocyte quality. Therefore, the melatonin levels in the follicular fluid as highly relevant biochemical markers of both oocyte quantity and quality can act as a good predictor of ovarian reserve as well as IVF outcomes.

In contrast with other markers of ovarian reserve, melatonin is a stable compound produced endogenously and also occurs naturally in some food. Both acute and chronic toxicity of melatonin is reported to be extremely low; no adverse effects were found when 1-300 mg or one gram melatonin was administered to human volunteers daily for 30 days (Jahnke et al. 1999, Vijayalaxmi et al. 2004). Melatonin has frequently been given to promote sleep as a dietary supplement (Jahnke et al. 1999). Despite great advances in IVF procedures, poor ovarian reserve remains a profound problem for female infertility. So the efficacy of administration of melatonin to the patients with poor ovarian reserve by which the intrafollicular melatonin levels appear to raise at the same time (Tamura et al. 2008) and supplementation of melatonin to their embryo culture medium or oocyte mature medium, as a new strategy, can be validated by more clinical trials.

In addition, we did not analyze the pregnancy rate because the fresh embryo transfer in poor ovarian response group was canceled due to the thin endometrium or insufficient embryos. We did not calculate cleavage rate either because of the mixture of IVF and ICSI procedures in our study. Other limitations of our study cannot be ignored. For example, the melatonin levels in follicular fluid were calculated by multiplying the melatonin value in single follicle by the number of oocytes retrieved. This calculation may have contributed to some bias if the concentration of melatonin in each preovulatory follicle was different. We attempted to minimize such bias by extracting fluids from follicles more than $19 \mathrm{~mm}$ in diameter and excluding blood-tinged or diluted fluids according to other investigators' strategy where the correlation coefficient between preovulatory follicular melatonin and follicular volume was not statistically significant (Brzezinski et al. 1987).

In conclusion, we show for the first time that the melatonin levels in follicular fluid are associated with both quantity and quality of oocytes and can predict IVF outcomes and can act as highly relevant biochemical markers of ovarian reserve. However, further large-scale, prospective studies are needed to validate our results.

\section{Declaration of interest}

The authors declare that there is no conflict of interest that could be perceived as prejudicing the impartiality of the research reported.

\section{Funding}

This work was supported by the National Natural Science Foundation of China (NSFC, 31172040, 31471399) to $\mathrm{C} Z$, by the Major Program of National Natural Science Foundation of China (81490743) to Z-J C, by the NSFC (81370692) to W P Li and by the Shanghai Commission of Science and Technology (12DZ2260600).

\section{Acknowledgements}

The authors express their appreciation to Dr Bruce D Murphy (University of Montreal) for critical reading of the manuscript and valuable advice and thank all of the patients for their participation in this study.

\section{References}

Abdalla H \& Thum MY 2004 An elevated basal FSH reflects a quantitative rather than qualitative decline of the ovarian reserve. Human Reproduction 19 893-898. (doi:10.1093/humrep/deh141) 
Ali AA, Bilodeau JF \& Sirard MA 2003 Antioxidant requirements for bovine oocytes varies during in vitro maturation, fertilization and development. Theriogenology 59 939-949. (doi:10.1016/S0093-691X(02)01125-1)

Aydın GA, Yavuz A, Terzi H \& Kutlu T 2015 Assessment of the relationship of basal serum anti-mullerian hormone levels with oocyte quality and pregnancy outcomes in patients undergoing ICSI. International Journal of Reproductive BioMedicine 13 231-236.

Balaban B, Urman B, Alatas C, Mercan R, Aksoy S \& Isiklar A 2001 Blastocyst-stage transfer of poor-quality cleavage-stage embryos results in higher implantation rates. Fertility and Sterility 75 514-518. (doi:10.1016/S0015-0282(00)01756-8)

Bancsi LsFJMM, Broekmans FJM, Eijkemans MJC, de Jong FH, Habbema JDF \& te Velde ER 2002 Predictors of poor ovarian response in in vitro fertilization: a prospective study comparing basal markers of ovarian reserve. Fertility and Sterility 77 328-336. (doi:10.1016/S00150282(01)02983-1)

Brzezinski A, Seibel MM, Lynch HJ, Deng MH \& Wurtman RJ 1987 Melatonin in human preovulatory follicular fluid. Journal of Clinical Endocrinology and Metabolism 64 865-867. (doi:10.1210/jcem-64-4865)

Chao H-T, Lee S-Y, Lee H-M, Liao T-L, Wei Y-H \& Kao S-H 2005 Repeated ovarian stimulations induce oxidative damage and mitochondrial DNA mutations in mouse ovaries. Annals of the New York Academy of Sciences 1042 148-156. (doi:10.1196/annals.1338.016)

Chen C-Q, Fichna J, Bashashati M, Li Y-Y \& Storr M 2011 Distribution, function and physiological role of melatonin in the lower gut. World Journal of Gastroenterology 17 33888-33898. (doi:10.3748/wjg.v17. i34.3888)

Dehghani-Mohammadabadi $M$, Salehi M, Farifteh F, Nematollahi S, Arefian E, Hajjarizadeh A, Parivar K \& Nourmohammadi Z 2014 Melatonin modulates the expression of BCL-xl and improve the development of vitrified embryos obtained by IVF in mice. Journal of Assisted Reproduction and Genetics 31 453-461. (doi:10.1007/s10815014-0172-9)

Ferraretti AP, Marca AL, Fauser BCJM, Tarlatzis B, Nargund G \& Gianaroli L 2011 ESHRE consensus on the definition of 'poor response' to ovarian stimulation for in vitro fertilizaiton: the Bologna criteria. Human Reproduction Update 26 1616-1624. (doi:10.1093/humrep/der092)

Gardner DK, Phil D, Lane M, Stevens J, Schlenker T \& Schoolcraft WB 2000 Blastocyst score affects implantation and pregnancy outcome: towards a single blastocyst transfer. Fertility and Sterility 73 1155-1158. (doi:10.1016/S0015-0282(00)00518-5)

Goud AP, Goud PT, Diamond MP, Gonik B \& Abu-Soud HM 2008 Reactive oxygen species and oocyte aging: Role of superoxide, hydrogen peroxide, and hypochlorous acid. Free Radical Biology and Medicine $\mathbf{4 4}$ 1295-1304. (doi:10.1016/j.freeradbiomed.2007.11.014)

He Y-M, Deng H-H, Shi M-H, Bodinga BM, Chen H-L, Han Z-S, Jiang Z-L \& Li Q-W 2016 Melatonin modulates the functions of porcine granulosa cells via its membrane receptor MT2 in vitro. Animal Reproduction Science 172 164-172. (doi:10.1016/j.anireprosci.2016.07.015)

Itoh MT, Ishizuka B, Kuribayashi Y, Amemiya A \& Sumi Y 1999 Melatonin, its precursors, and synthesizing enzyme activities in the human ovary. Molecular Human Reproduction 5 402-408. (doi:10.1093/ molehr/5.5.402)

Jahnke G, Marr M, Myers C, Wilson R, Travlos G \& Price C 1999 Maternal and developmental toxicity evaluation of melatonin administered orally to pregnant Sprague-Dawley rats. Toxicological Sciences 50 271-279. (doi:10.1093/toxsci/50.2.271)

Kang J-T, Koo O-J, Kwon D-K, Park H-J, Jang G, Kang S-K \& Lee B-C 2009 Effects of melatonin on in vitro maturation of porcine oocyte and expression of melatonin receptor RNA in cumulus and granulosa cells. Journal of Pineal Research 46 22-28. (doi:10.1111/j.1600079X.2008.00602.x)

Kauffman AS, Clifton DK \& Steiner RA 2007 Emerging ideas about kisspeptin-GPR54 signaling in the neuroendocrine regulation of reproduction. Trends in Neurosciences 30 504-511. (doi:10.1016/j. tins.2007.08.001)

Malpaux B, Migaud M, Tricoire H \& Chemineau P 2001 Biology of mammalian photoperiodism and the critical role of the pineal gland and melatonin. Journal of Biological Rhythms 16 336-347. (doi:10.1177/07 4873001129002051)

Manchester LC, Coto-Montes A, Boga JA, Andersen LPH, Zhou Z, Galano A, Vriend J, Tan D-X \& Reiter RJ 2015 Melatonin: an ancient molecule that makes oxygen metabolically tolerable. Journal of Pineal Research 59 403-419. (doi:10.1111/jpi.12267)

Nakano M, Kato Y \& Tsunoda Y 2012 Effect of melatonin treatment on the developmental potential of parthenogenetic and somatic cell nucleartransferred porcine oocytes in vitro. Zygote 20 199-207. (doi:10.1017/ S0967199411000190)

Niknafs B, Mehdipour A \& Roushandeh AM 2014 Melatonin improves development of early mouse embryos impaired by actinomycin-D and TNF- $\alpha$. International Journal of Reproductive BioMedicine 12 799-804.

Qiao J, Ma C-H, Liu J-Y, Ma X, Li S-W, Yang Y-Z, Zhang B, La X-L, Wang X-H, Zhu Y-M, et al. 2015 A consensus of poor ovarian response. Reproduction and Contraception 35 211-223. (doi:10.7669/j.issn.0253357X.2015.04.0211)

Rad SS, Abbasalizadeh S, Haghjo AG, Sadagheyani M, Montaseri A \& Rad JS 2015 Evaluation of the melatonin and oxidative stress markers level in serum of fertile and infertile women. International Journal of Reproductive BioMedicine 13 439-444.

Reiter RJ, Tan D-X, Manchester LC, Paredes SD, Mayo JC \& Sainz RM 2009 Melatonin and Reproduction Revisited. Biology of Reproduction 81 445-456. (doi:10.1095/biolreprod.108.075655)

Reiter RJ, Tan D-X \& Fuentes-Broto L 2010 Melatonin: a multitasking molecule. Progress in Brain Research 181 127-151. (doi:10.1016/ s0079-6123(08)81008-4)

Reiter RJ, Rosales-Corral SA, Manchester LC \& Tan D-X 2013 Peripheral reproductive organ health and melatonin: ready for prime time. International Journal of Molecular Sciences 14 7231-7272. (doi:10.3390/ ijms14047231)

Reiter RJ, Tamura H, Tan DX \& Xu X-Y 2014 Melatonin and the circadian system: contributions to successful female reproduction. Fertility and Sterility 102 321-328. (doi:10.1016/j.fertnstert.2014.06.014)

Rodriguez C, Mayo JC, Sainz RM, Antolin I, Herrera F, Martin V \& Reiter RJ 2004 Regulation of antioxidant enzymes: a significant role for melatonin. Journal of Pineal Research 36 1-9. (doi:10.1046/j.1600079X.2003.00092.x)

Sakaguchi K, Itoh MT, Takahashi N, Tarumi W \& Ishizuka B 2012 The rat oocyte synthesises melatonin. Reproduction, Fertility and Development 25 674-682. (doi:10.1071/RD12091)

Smeenk JMJ, Sweep FCGJ, Zielhuis GA, Kremer JAM, Thomas CMG \& Braat DDM 2007 Antimüllerian hormone predicts ovarian responsiveness, but not embryo quality or pregnancy, after in vitro fertilization or intracyoplasmic sperm injection. Fertility and Sterility $\mathbf{8 7}$ 223-226. (doi:10.1016/j.fertnstert.2006.06.019)

Stadtman ER 2002 Importance of individuality in oxidaive stress and aging. Free Radical Biology and Medicine 33 597-604. (doi:10.1016/S08915849(02)00904-8)

Tamura H, Takasaki A, Miwa I, Taniguchi1 K, Maekawa R, Asada H, Taketani T, Matsuoka A, Yamagata Y, Shimamura K, et al. 2008 Oxidative stress impairs oocyte quality and melatonin protects oocyte from free radical damage and improves fertilization rate. Journal of Pineal Research 44 280-287. (doi:10.1111/j.1600-079X.2007.00524.X)

Tamura H, Nakamura Y, Korkmaz A, Manchester LC, Tan D-X, Sugino N \& Reiter RJ 2009 Melatonin and the ovary: physiological and pathophysiological implications. Fertility and Sterility 92 328-343. (doi:10.1016/j.fertnstert.2008.05.016)

Tamura H, Takasaki A, Taketani T, Tanabe M, Kizuka F, Lee L, Tamura I, Maekawa R, Aasada H, Yamagata Y, et al. 2012 The role of melatonin as an antioxidant in the follicle. Journal of Ovarian Research 55. (doi:10.1186/1757-2215-5-5)

Tan D-X, Manchester LC, Terron MP, Flores LJ \& Reiter RJ 2007 One molecule, many derivatives: a never-ending interaction of melatonin with reactive oxygen and nitrogen species? Journal of Pineal Research 42 28-42. (doi:10.1111/j.1600-079X.2006.00407.x)

Tomás-Zapico C \& Coto-Montes A 2005 A proposed mechanism to explain the stimulatory effect of melatonin on antioxidative enzymes. Journal of Pineal Research 39 99-104. (doi:10.1111/j.1600-079x.2005.00248.x)

Touitou Y 2001 Human aging and melatonin. Clinical relevance. Experimental Gerontology 36 1083-1100. (doi:10.1016/S05315565(01)00120-6)

Vakkuri O, Kivela A, luoto JL, Valtonen M \& Kauppila A 1996 Decrease in melatonin precedes follicle-stimulating hormone increase during perimenopause. European Journal of Endocrinology 135 188-192. (doi:10.1530/eje.0.1350188) 
Vijayalaxmi, Reiter RJ, Tan D-X, Herman TS \& Thomas CR Jr 2004 Melatonin as a radioprotective agent: a review. International Journal of Radiation Oncology, Biology, Physics 59 639-653. (doi:10.1016/j. ijrobp.2004.02.006)

Waldhauser F, Weiszenbacher G, Tatzer E, Gisinger B Waldhauser M, Schemper M \& Frisch H 1988 Alterations in nocturnal serum melatonin levels in humans with growth and aging. Journal of Clinical Endocrinology and Metabolism 66 648-652. (doi:10.1210/ jcem-66-3-648)

Walecka-Kapica E, Chojnacki J, Stepien A, Wachowska-Kelly P, Klupinska G \& Chojnacki C 2015 Melatonin and female hormone secretion in postmenopausal overweight women. International Journal of Molecular Sciences 16 1030-1042. (doi:10.3390/ijms16011030)

Wang X, Falcone T, Attaran M, Goldberg JM, Agarwal A \& Sharma RK 2002 Vitamin C and Vitamin E supplementation reduce oxidative stressinduced embryo toxicity and improve the blastocyst development rate. Fertility and Sterility $\mathbf{7 8}$ 1272-1277. (doi:10.1016/S00150282(02)04236-X)

Wang F, Tian X, Zhang L, Tan D, Reiter RJ \& Liu G 2013 Melatonin promotes the in vitro development of pronuclear embryos and increases the efficiency of blastocyst implantation in murine. Journal of Pineal Research 55 267-274. (doi:10.1111/jpi.12069)
Weenee C, Laven JSE, Bergh ARMV, Cranfield M, Groome NP, Visser JA, Kramer P, Fauser BCJM \& Themmen APN 2004 Anti-Mullerian hormone expression pattern in the human ovary: potential implications for initial and cyclic follicle recruitment. Molecular Human Reproduction $\mathbf{1 0}$ 77-83. (doi:10.1093/molehr/gah015)

Wiener-Megnazi Z, Vardi L, Lissak A, Shnizer S, Reznick AZ, Ishai D, Lahav-Baratz S, Shiloh H, Koifman M \& Dirnfeld M 2004 Oxidative stress indices in follicular fluid as measured by the thermochemiluminescence assay correlate with outcome parameters in in vitro fertilization. Fertility and Sterility 82 (Supplement 3) 1171-1176. (doi:10.1016/j.fertnstert.2004.06.013)

Zhang X, Li X-H, Ma X, Wang Z-H, Lu S \& Guo Y-L 2006 Redox-induced apoptosis of human oocytes in resting follicles in vitro. Journal of the Society for Gynecologic Investigation 13 451-458. (doi:10.1016/j. jsgi.2006.05.005)

Received 1 October 2016

First decision 8 November 2016

Revised manuscript received 26 November 2016

Accepted 6 January 2017 